\title{
EL SERVICIO DE DELIVERY COMO ESTRATEGIA COMPETITIVA
}

DELIVERY SERVICE AS COMPETITIVE STRATEGY

Johannes Schmidt Urdanivia*

unmsmfca@gmail.com

Hugo Alberto Young Gonzales***

hugo_young@hotmail.com

Sergio Podestá Cuadros******

spodestac@gmail.com

[RECEPCIÓN: MARZO DE 2015 / CONFORMIDAD: ABRIL DE 2015]

\section{RESUMEN}

En la actualidad, los empresarios en el Perú se han visto en la obligación de utilizar estrategias que permitan crear valor en sus organizaciones y ser competitivos en un mercado cada vez más dinámico, utilizando dentro de estas estrategias, el servicio delivery que ha generado todo un cambio en la forma de interactuar entre empresa-cliente, cambiado el modelo de negocios de muchas compañías y teniendo como resultado beneficios para ambas partes. Este artículo analiza la ejecución del delivery en empresas significativas del medio, mostrando las principales características de esta estrategia y las ventajas de su aplicación.

Palabras clave: Delivery, estrategia, competitividad y beneficios.

\begin{abstract}
Currently, managers in Peru have been obliged to use strategies to create value in their organizations and be competitive in a dynamic market, using the delivery service as a means of changing the business-customer relationship, changing the business model of many companies and obtaining benefits for both parties. This article discusses the delivery in important companies, showing the main features of this strategy and the benefits of its application.
\end{abstract}

Keywords: Delivery, strategy, competitiveness and profits.

* Maestría en Administración con Mención en Gestión Empresarial (UNMSM). Estudios de Administración (Universidad de Buenos Aires). Profesor ordinario de la Facultad de Ciencias Administrativas (UNMSM). Administrador en el Colegio de Psicólogos del Perú (Consejo Directivo Regional I - Lima).

*** Maestría Profesional en Gestión de Negocios Internacionales (UNMSM) y Grado de Master Droit, Economie, Gestión (Université Bordeaux IV) con especialidad en Management International. Licenciado en Administración (UNMSM). Especialización en Finanzas. Docente en la Universidad Nacional Mayor de San Marcos y la Universidad Peruana de Ciencias Aplicadas (UPC). Ex funcionario en BBVA Continental y actual funcionario en COFIDE. Consultor en Finanzas.

***** Licenciado en Administración (UNMSM), estudios de maestría en Ordenamiento y Gestión Ambiental (UNMSM). Especialista en Administración Hotelera por CENFOTUR, consultor en Calidad Turística y Turismo Rural Comunitario del MINCETUR, Docente Permanente en la Facultad de Ciencias Administrativas de la UNMSM. 


\section{INTRODUCCIÓN}

La búsqueda y logro de la competitividad es imperativo para las organizaciones. Para este propósito las compañías encuentran en el servicio de delivery la posibilidad de ampliar su capacidad de atención al público, una forma de acercarse a los clientes, un mayor posicionamiento de su marca y una alternativa de incrementar las ganancias.

Para algunas empresas, en sus inicios, el delivery les permite atender un mercado sin necesariamente contar con un local de atención, apoyándose en los pedidos telefónicos. Castillo (2014, p. 86) en "Plan de negocios para la puesta en marcha de un fast food saludable en la ciudad de Piura", sostiene: “... como estrategia de distribución que es propicia a nuestro modelo de servicio es la distribución directa, es decir nosotros nos encargamos de la preparación de los alimentos que se ofrecerán y nosotros mismos somos los encargados de hacerlos llegar al público en el punto de venta”.

Para las empresas consolidadas, atender por delivery es un signo de compromiso de servicio, llegando incluso a fijarse estándares de llegada al cliente o -en caso de incumplimiento del plazo de entrega- la entrega del pedido sin cobro al cliente.

Sin embargo, cuando las obras públicas en las ciudades generan congestionamiento, la rapidez de la entrega se puede ver afectada. Flores (2004, p. 28) en "Optimización en la entrega de productos para una cadena de abastecimientos" (Informe para optar el título de ingeniero industrial) indica que la determinación de los esquemas de delivery óptimo, es en general un problema muy dificultoso, compitiendo en dificultad con el job shop (que considera pequeños pedidos, teniendo en cuenta las especificidades solicitadas por el cliente) que asigna trabajos a máquina, teniendo cada trabajo el orden tecnológico diferente.

La creciente adopción del sistema de delivery por parte de empresas de diversos tipos llevó a analizar esta modalidad de servicio, su participación en las ganancias de las empresas y la satisfacción de los consumidores.

En otros ámbitos y dados los avances de la tecnología se empieza a pensar en la legislación para autorizar el delivery empleando drones como ya lo ha hecho un grupo de estudiantes de la Universidad de Berlín, en Alemania tal como dice Oppenheimer A. (2014, p. 24) en "iCrear o morir!, La esperanza de América Latina y las cinco claves de la innovación": "El profesor Rojas González, que enseña inteligencia artificial en esa universidad alemana, me comentó que la tecnología para repartir pizzas, medicamentos o cualquier otro producto liviano ya existe y se está usando... varias empresas de envíos a domicilio, como Federal Express y UPS entre otras, ya están contemplando el sistema y esperando la luz verde de las autoridades para entrar en acción".

La demanda de rapidez en un mundo apresurado augura al servicio de delivery un promisorio y competitivo futuro. En tal sentido, este artículo tiene como objetivo principal dar a conocer la utilización del servicio delivery, sus características y aplicaciones reales como estrategia que incrementa la competitividad en las empresas.

\section{MÉTODOS}

Los principales métodos y técnicas de recolección de información que se aplicaron en la presente investigación fueron los siguientes:

Entrevistas personales:

- Se entrevistaron a los administradores de locales seleccionados de las siguientes empresas: Tortas Gaby, Botica Perfumería Inkafarma, Pizza Papa John's, Pollería Norky's, Sanguchería Pasquale Hnos. y Sanguchería La Lucha.

Cuestionarios estructurados:

- Se desarrollaron cinco cuestionarios estructurados para aplicarlos al personal del servicio de delivery, como también a los administradores de los locales seleccionados.

Observación:

- Se tomaron fotos en las etapas del proceso de delivery de cada empresa en la que se entrevistó al administrador.

Fuentes secundarias:

- Se analizó información bibliográfica sobre el servicio de delivery.

- Se analizaron las páginas web de las empresas analizadas.

\section{RESULTADOS Y DISCUSIÓN}

A continuación se muestra el análisis sobre las empresas seleccionadas en este estudio, que 
utilizan el sistema de delivery como estrategia para incrementar su competitividad.

Tortas Gaby ha sido premiada durante 10 años consecutivos con el importante galardón Cinta de Oro a "La Mejor del Año", asimismo con el premio

\section{SERVICIO DE DELIVERY: TORTAS GABY}

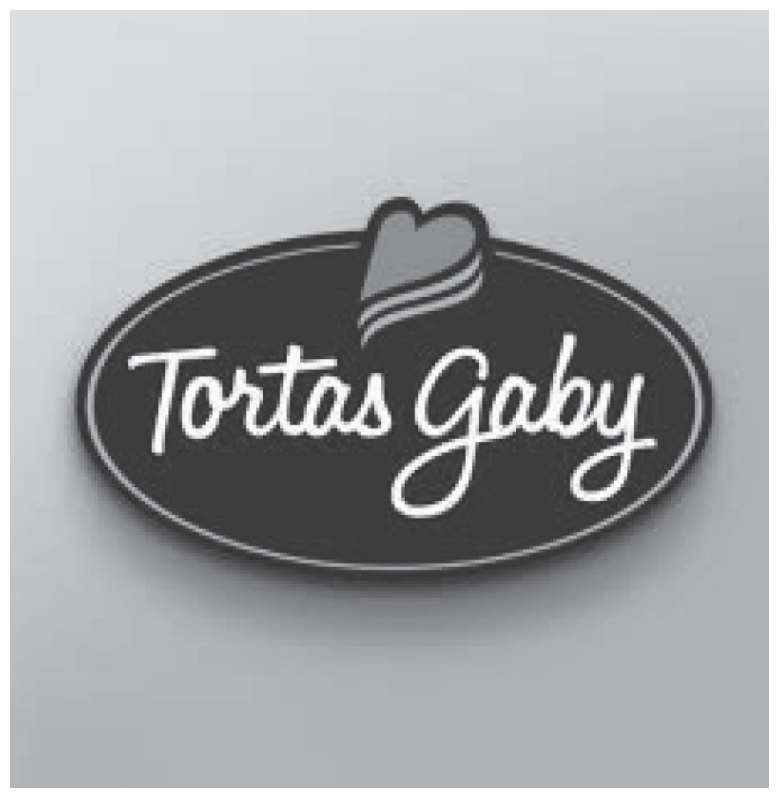

"Manos PYME del Perú" otorgado por el Instituto Nacional de Defensa de la Competencia y de la Protección de la Propiedad Intelectual (INDECOPI) por el posicionamiento de marca y la calidad de los productos y atención. Por lo tanto, estos reconocimientos y en especial el del público que día a día les acompaña, son su mayor aliciente y también un reto constante que los compromete a seguir adelante ofreciendo cada vez una mejor experiencia de compra a sus clientes. Por esta razón se detallarán algunas características de su servicio de delivery:

El proceso del servicio de delivery se inicia con el pedido del cliente que puede ser vía on line pero deben realizarse con un mínimo de 48 horas de anticipación; sin embargo, si el cliente necesita que su pedido llegue cuanto antes pueden utilizar la página web como catálogo, luego se comunican a la central telefónica para que le tomen el pedido y registren sus datos en una guía que será entregada al encargado de llevar el pedido y otra se queda en la empresa para archivarla. Al momento de recibir la llamada se aseguran de que el producto requerido se encuentre disponible, acuerdan la hora de entrega y también la forma de pago, que puede ser en efectivo contra entrega y/o pagos con tarjeta de crédito o débito o vía on line a través de la página web.

Dentro de las mediciones relacionadas con el cliente, la empresa se preocupa por la calidad del servicio y tiempo de entrega. Sin embargo, para prevenir las pérdidas, la empresa incluye cláusulas en sus contratos en las que el cliente se hace responsable de cualquier inconveniente; por ejemplo: si la movilidad llega dentro del rango de tiempo indicado para la entrega del pedido y en el lugar no hubiere quien pueda cancelarlo o recibirlo, el delivery esperará un plazo máximo de 10 minutos, luego procederán a dejar una nota informativa en la que se le solicitará contactarse con ellos a fin de recoger el pedido en las instalaciones de la empresa, debiendo cancelar el servicio de movilidad. En el caso de preferir un reenvío, el cliente asumirá también la segunda movilidad.

Para esta empresa, el concepto de La Lucha, significa el esfuerzo y dedicación para alcanzar las metas y objetivos que sirvieron de inspiración

SERVICIO DE DELIVERY: SANGUCHERIA LA LUCHA

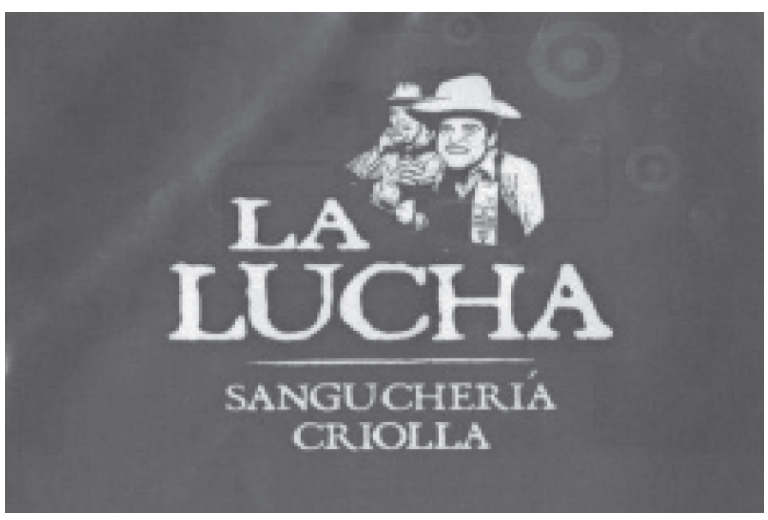

para el nombre de su empresa, y es que consideran que la voluntad diaria de los peruanos para alcanzar sus sueños y proyectos de vida, es digna de reconocer. Además, reconocen la trayectoria de nuestra gastronomía y de las tradiciones que son parte de la cultura peruana, es por ello que procuran fusionar los sanguches criollos con un ambiente muy tradicional, cuidando cada detalle para hacer sentir al cliente como en su propio hogar. Respecto a su servicio de delivery, es relevante para la empresa porque contribuye con su nivel de ventas, fideliza a los clientes, y también es una manera importante de realizar publicidad. El proceso del servicio de delivery se detalla en la Figura $\mathrm{N}^{\circ} 1$, el cual cuenta con el personal especializado para realizar el trabajo de recepción del pedido y distribución de los sanguches. 


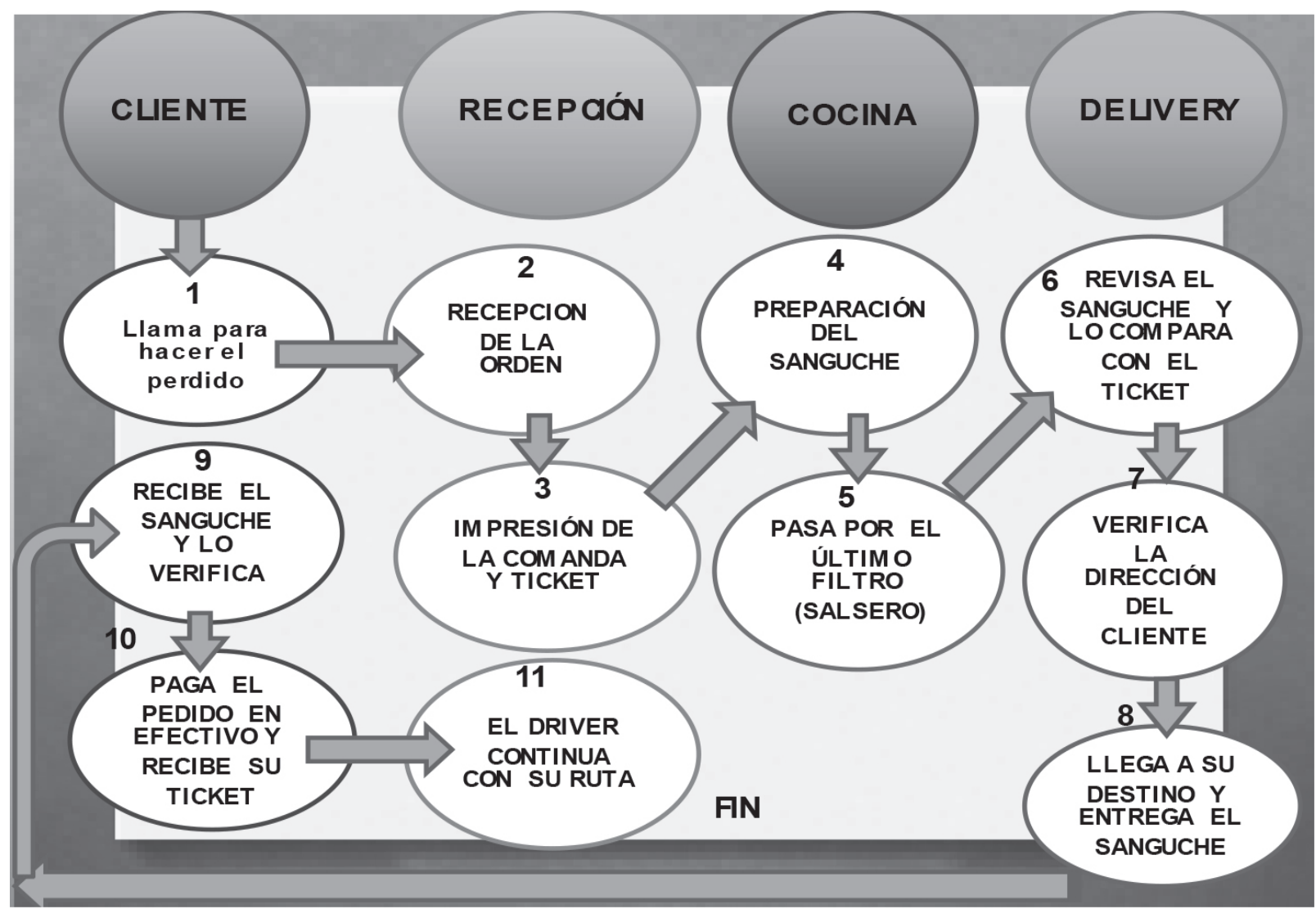

Fig. $\mathrm{N}^{\mathrm{O}}$ 1: Proceso del servicio de delivery

Como su enfoque del servicio de delivery, es darle valor al cliente, tratan de ser los más responsables posibles en el tiempo de entrega del producto acordado con el cliente y también de entregar el producto adecuadamente, por esta razón se tiene mucho cuidado con el traslado de los sanguches. Sin embargo, si se comete algún error involuntario o hay una queja de los clientes, se procede a entregar vales de consumo.

Papa John's Perú es una franquicia adquirida por la Corporación Peruana de Restaurantes (CORPERES). La primera tienda en Perú fue aperturada

\section{SERVICIO DE DELIVERY: PAPA JHON'S}

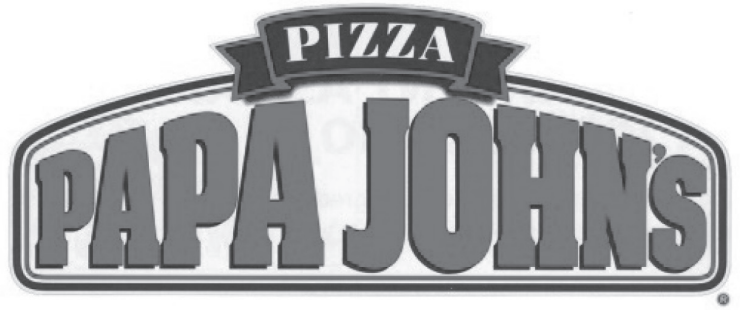

en el 2004, desde entonces han crecido progresivamente. Actualmente Papa John's Perú cuenta con varias tiendas en Lima y en provincia. Su visión es ser una corporación líder a nivel internacional, ofreciendo productos y un servicio de calidad superior. Papa John's cuenta con los siguientes premios y reconocimientos: Mejor Franquiciatario Internacional del año 2006, Medalla de Oro a la Excelencia del año 2006, Mejor Food \& Beverage 2006 en el Aeropuerto Internacional Jorge Chávez, número 1 en satisfacción al cliente 1999-2006, Mejor Franquicia Regional del año 2010, Mejor Marketing Regional del año 2010, Premio a la Calidad 2010, Premios a la excelencia en delivery 2010. En tal sentido, habiendo recibido tantos reconocimientos y uno de ellos a la excelencia en el servicio de delivery, detallaremos algunas de sus fortalezas en el servicio:

- El servicio de delivery para todas las pizzerías de Papa John's no es mediante una central telefónica, cada tienda tiene diferente número telefónico y esto se debe a que desean tener una relación más personalizada con los clientes, lo que permite contar con una base de datos de los clientes por área geográfica, gustos y preferencias.

- Una de sus fortalezas principales son las personas que trabajan en la empresa, por- 
que las consideran como el principal activo que tienen, ya que agregan inteligencia a su negocio. Por lo tanto, gracias a la fortaleza referida y a la implementación de los procesos de calidad han podido elevar la calidad de sus productos, productividad y competitividad en el mercado.

- También cuentan con una etapa de postventa en su proceso del delivery con la finalidad de tomar medidas correctivas en el caso que sea necesario, es por ello que permanentemente, ya sea por teléfono y/o el mismo driver miden el grado de satisfacción de los clientes en cuanto al tiempo de entrega del producto, cortesía del personal, calidad de las pizzas, limpieza de los materiales y otros.

- Para la distribución de los productos utilizan motos lineales modernas y se les adecúa en la parte posterior una caja donde se organizan mejor los productos y también la parte externa sirve como medio publicitario; así mismo el personal motorizado está calificado y utiliza el uniforme de Papa John's.

- El servicio de delivery no tiene recargo alguno y si no llega antes de la media hora, el pedido es totalmente gratis, además se le da al cliente un vale por otro pedido igual que lo puede consumir solo en la tienda a donde llamó a hacer su pedido de delivery, esto es una garantía de calidad que otorga Papa John's por la demora.

\section{SERVICIO DE COURIER}

La experiencia empresarial nos demuestra que diversas organizaciones e instituciones dependen de sus clientes y, por lo tanto, se esfuerzan en sobrepasar sus expectativas, cumplir con los requisitos y comprender sus necesidades actuales y futuras. Es decir, la administración está orientada a la calidad, servicio, oportunidad de la entrega y a la capacidad de responder a los deseos del cliente. En tal sentido, las empresas independientemente de su tamaño y actividad son conscientes que un factor crítico de éxito es el de trasladar con mayor eficiencia y eficacia a sus clientes los productos que ya fueron comprados y/o adquiridos, para ello están utilizando intermediarios como son los "servicios de courier", los cuales realizan el envío de paquetes o documentos en un tiempo determinado. Las características básicas que tiene el servicio de courier son la velocidad, ya que en un tiempo determinado se tiene que enviar un documento o paquete; también la seguridad es importante en este servicio, ya que el cliente debe confiar que su envío llegará al destino previsto; la empresa que ofrece este servicio de courier deberá hacer un seguimiento y mantener informado a su cliente sobre el estado de su envío y cumplir con el plazo de entrega establecido para generar confianza y fidelizar al cliente. Es necesario tener en cuenta que el servicio de courier también presenta restricciones legales y/o administrativas para el traslado de mensajería o paquetes. La mayoría de empresas que ofrecen el servicio de courier cuentan con capacidad operativa para ofrecer servicios especiales como entrega de encomiendas, entrega en 24 horas, entrega urgente y entrega internacional. También pueden ofrecer servicios corporativos como almacenaje, impresión de papelería, cobranza, entre otros.

\section{CONCLUSIONES}

1. De la investigación realizada podemos concluir que el servicio delivery es una estrategia que beneficia tanto a las empresas que la utilizan como a los clientes en los siguientes aspectos:

Para las empresas:

- Incremento en el número de transacciones comerciales (pedidos y clientes).

- Aumenta la facturación de la empresa.

- Reduce costos.

- Acercamiento de la empresa al cliente.

- Utilización de los medios de comunicación y de distribución para promociones y publicidad.

Para los clientes:

- Evita el desplazamiento físico al lugar de compra.

- Ahorro de tiempo y dinero.

- Mayor comodidad para realizar la compra.

- Utilización de la tecnología para el pago.

- Varias formas de pago (efectivo, tarjetas de crédito y débito e Internet).

Asimismo, la investigación realizada ha arrojado que en la mayoría de los locales analizados, 
este servicio representa el $40 \%$ de sus ganancias, que se han incrementado los ingresos desde su implementación y que ha generado una especialización en el tema.

Las empresas adecuan este servicio a su modelo de negocio, obligándose a invertir lo necesario para su óptimo funcionamiento e innovar de forma continua debido a las mayores exigencias de los clientes y a la lucha continua con la competencia, dejando un gran reto para el futuro.

\section{LITERATURA CITADA}

Castillo, L. (2014). Plan de negocios para la puesta en marcha de un fast food saludable en la ciudad de Piura. Universidad de Piura, Piura.

Flores, M. (2004). Optimización en la entrega de productos para una cadena de abastecimientos (Informe para optar el Título de Ingeniero Industrial). UNMSM, Lima.

Oppenheimer, A. (2014). iCrear o morir!, la esperanza de América Latina y las cinco claves de la innovación. Editorial Debate, Barcelona. 